\title{
Use of computed tomography for assessing bone mineral density
}

\author{
Joseph J. Schreiber, M.D., ${ }^{1}$ Paul A. Anderson, M.D., ${ }^{2}$ and Wellington K. Hsu, M.D. ${ }^{3}$ \\ ${ }^{1}$ Department of Orthopaedic Surgery, Hospital for Special Surgery, New York, New York; ${ }^{2}$ Department \\ of Orthopedics \& Rehabilitation, University of Wisconsin, Madison, Wisconsin; and ${ }^{3}$ Department of \\ Orthopaedic Surgery, Northwestern University Feinberg School of Medicine, Chicago, Illinois
}

\begin{abstract}
Assessing local bone quality on CT scans with Hounsfield unit (HU) quantification is being used with increasing frequency. Correlations between $\mathrm{HU}$ and bone mineral density have been established, and normative data have been defined throughout the spine. Recent investigations have explored the utility of HU values in assessing fracture risk, implant stability, and spinal fusion success. The information provided by a simple HU measurement can alert the treating physician to decreased bone quality, which can be useful in both medically and surgically managing these patients.
\end{abstract}

(http://thejns.org/doi/abs/10.3171/2014.5.FOCUS1483)

\section{KEY WoRds • Hounsfield unit • osteoporosis • bone mineral density}

$\mathrm{W}$ ITH an aging population, osteoporosis is increasingly becoming a public health concern. Recent estimates suggest that more than 10 million people are affected in the US, with an additional 44 million citizens at risk. ${ }^{11}$ The disease is typically characterized by an age-related reduction in bone strength that predisposes affected individuals to low-energy fractures. In the spine, the incidence of osteoporotic vertebral fractures exceeds 1.4 million events annually. ${ }^{13}$ Osteoporotic fractures are a significant cause of pain, disability, and decreased quality of life, which are associated with substantial health care costs exceeding $\$ 17$ billion (US dollars) annually. ${ }^{3}$

Dual x-ray absorptiometry (DXA) is currently the standard for assessing bone mineral density (BMD) and has been correlated with fracture risk and treatment efficacy. ${ }^{21}$ While useful for assessing osteopenia or osteoporosis, ${ }^{1}$ it is not without methodological limitations. ${ }^{37}$

The use of Hounsfield units (HUs) from CT scanning to assess regional BMD of the spine has recently been described, ${ }^{30}$ with several subsequent studies exploring its utility in assessing fracture risk and prognosticating fusion success. As described by Pickhardt et al., when CT scans are obtained for other clinical indications, they may also be used for "opportunistic screening for osteoporosis." ${ }^{27}$

Hounsfield unit values are a measurement of the standardized linear attenuation coefficient of tissue, based on a defined scale of 0 for water and -1000 for air. Modern radiology imaging software programs allow this to be

\footnotetext{
Abbreviations used in this paper: $\mathrm{BMD}=$ bone mineral density; DXA = dual $\mathrm{x}$-ray absorptiometry; $\mathrm{HU}=$ Hounsfield unit; $\mathrm{ROI}=$ region of interest.
}

calculated from a region of interest (ROI) on CT scans without any additional cost or radiation exposure. Values are calculated based on the following formula: $\mathrm{HU}=([\mu$ $\left.-\mu_{\mathrm{w}}\right]\left(\mu_{\mathrm{w}}\right) \times 1000$, where $\mu$ is defined as the linear x-ray attenuation coefficient of the selected voxel and $\mu_{\mathrm{w}}$ the attenuation coefficient of distilled water at room temperature and pressure.

With the advent of automated exposure control on modern CT scanners, the calibrating phantoms that were historically used for quantitative $\mathrm{CT}$ are not required. ${ }^{10,19,30}$ This technology uses real-time detector feedback and data from the scout view to adjust tube current and exposure time. Tissue density and patient body habitus are accounted for, thereby producing a more homogeneous energy spectrum, shortening radiation exposure time, and resulting in more accurate $\mathrm{HU}$ values of the targeted tissue.

Important differences in measurements of BMD between DXA and CT should be considered (Table 1). Dual $\mathrm{x}$-ray absorptiometry is a planar measurement of density expressed as grams of mineral per square centimeter scanned $\left(\mathrm{g} / \mathrm{cm}^{2}\right)$, while the values obtained from CT scans are volumetric $\left(\mathrm{g} / \mathrm{mm}^{3}\right)$. Dual x-ray absorptiometry includes the posterior elements of the spine, and therefore may be inaccurate or not possible in cases of severe spinal degeneration, scoliosis, or following lumbar surgery. Computed tomography techniques can be limited to specific regions of interest, such as the vertebral body trabeculae, which has been shown to be most predictive of fracture. ${ }^{9}$

One of the disadvantages of using HU values to estimate bone quality is the lack of clinical support compared with DXA. Bone quality assessment is usually done using a DXA T-score, which is defined by the $\mathrm{WHO}$ as a value 
TABLE 1: Comparison of DXA and CT for assessing spinal bone mineral density

\begin{tabular}{lll}
\hline \multicolumn{1}{c}{ Parameter } & DXA & CT (HU) \\
\hline radiation dose & low & high except when used opportunistically \\
cost & $\$ 200-250$ & none when used opportunistically \\
ROI & includes cortex, trabecular bone, pedicles, posterior elements & any specific ROI \\
units & planar $\left(\mathrm{g} / \mathrm{cm}^{2}\right)$ & volumetric $\left(\mathrm{g} / \mathrm{mm}^{3}\right)$ \\
acceptance & gold standard & novel \\
contraindications & lumbar surgery, severe spinal degeneration, scoliosis & none \\
\hline
\end{tabular}

corresponding to the number of standard deviations that an individual's BMD differs from that of a young, healthy control as follows: ${ }^{1}$ T-score $=\left(\mathrm{BMD}_{\text {(patient) }}-\mathrm{BMD}_{\text {(reference) }}\right) /$ $\mathrm{SD}_{\text {(reference) }}$, where $\mathrm{BMD}_{\text {(reference) }}$ and $\mathrm{SD}_{\text {(reference) }}$ are bone mineral density and its standard deviation of healthy controls in their $20 \mathrm{~s}$, respectively.

It is the T-score, rather than the BMD or HU, that is used to both diagnose and measure treatment efficacy, as precise parameters based on HUs not yet readily available. T-score evaluations are somewhat limited in clinical utility, as the majority of patients who sustain fragility fractures are not in the osteoporotic range. ${ }^{14}$

The purpose of this paper is to review the reliability and validity of the techniques used to estimate bone health using CT HU measurements. We will also demonstrate examples of their practical use to identify patients at risk for osteoporosis and how these values could be used for surgical planning in cases of trauma, degeneration, and deformity.

\section{Methods}

\section{Technique for HU Assessment of the Spine}

Schreiber et al. described a simple technique for assessing regional BMD of the spine that was correlated with results of DXA scans as well as compressive strength in osseous models. ${ }^{30}$ Using standard picture archiving and communication system software, an ROI is drawn at 3 points on axial images that are obtained as parallel to the endplates as possible: just inferior to the superior endplate, midvertebral body, and just superior to the inferior endplate (Fig. 1). The ROI is drawn encapsulating only cancellous bone and avoiding cortical edges, osseous abnormalities, and voids such as vascular channels. The software calculates the average HU in the ROI for each image. An average of the 3 measurements determines the HU for individual vertebral levels. Others have found that a single midbody ROI is as reliable as the average of 3 measurements and have used an automated software program to generate values. ${ }^{26,27}$

\section{Study Cohorts}

All studies had institutional review board exemptions. Several patient cohorts were used to answer specific clinical questions. Twenty-five consecutive patients who underwent CT scanning to evaluate possible spinal injury and who had recently undergone DXA were used to evaluate reliability and correlations. Eighty patients who were evaluated for trauma were used to measure normative values. ${ }^{30}$ Twenty patients with adjacent-segment fractures after spinal fusion along with 20 matched nonfracture controls were used to assess the association between HU and adjacent-segment fracture. ${ }^{22}$ The CT scans of 28 patients with a combined 52 levels of lumbar interbody fusion were used to assess the association between $\mathrm{HU}$ and fusion success.

\section{Reliability of HU Measurements}

The intra- and interobserver reliability of HU measurements was performed using an interclass correlation coefficient. Two observers independently measured HU at 3 locations of 4 vertebrae in 25 subjects.

\section{Normative Data}

Normative data stratified by sex and decade of life were determined by Schreiber et al. ${ }^{30}$ based on CT scans
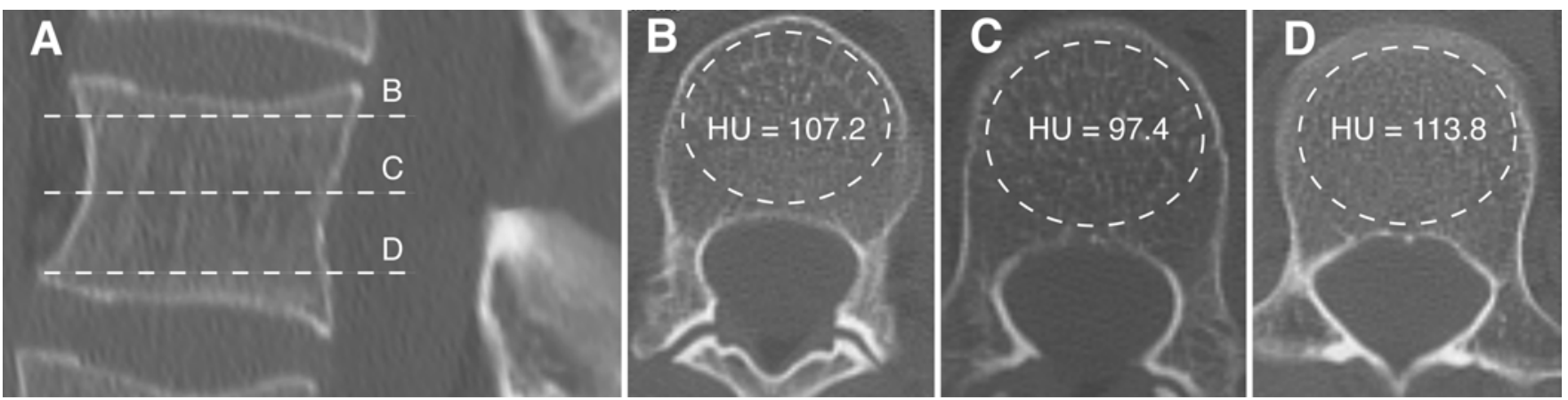

FIG. 1. Computed tomography scans illustrating the technique for calculating vertebral BMD with HUs. A: Sagittal slice of the L-3 vertebral body demonstrating axial planes of interest. B-D: Axial images showing $\mathrm{HU}$ values generated by the imaging software program. The mean value of B-D is used for calculating vertebral body bone density. 
from 80 consecutively presenting trauma patients grouped by decade from 10-19 years to $80-89$ years, with 10 patients in each decade. These data were compared with other published normative data. ${ }^{22}$

\section{Correlation of $H U$ and DXA}

Twenty-five patients undergoing lumbar CT and DXA within 6 months of each other were identified, and the BMD, T-score, and HU value were measured from L-1 to L-4. ${ }^{30}$ These patients had a mean age of 71.5 years with a range of 33-87 years. There were 18 female and 7 male patients. Correlation of T-score and BMD from DXA to HU measurement was performed using the Pearson correlation coefficient. Each patient was grouped into normal (T-score $\geq-1.0$ ), osteopenia (T-score -1.0 to -2.5 ), and osteoporotic (T-score $\leq-2.5$ ). The HU threshold values for each bone health state were reported as the mean and $95 \%$ confidence intervals.

\section{HU Value and Fracture Risk}

Meredith et al. matched 20 patients with adjacentsegment fractures after spinal fusion with nonfracture controls of similar age, sex, body mass index, and surgical procedure. ${ }^{22}$ Hounsfield unit measurements were taken throughout the spine from preoperative CT scans, and a Student t-test was used to compare means between the groups.

\section{HU Value and Fusion Success}

Schreiber et al. compared the HU of vertebral bodies adjacent to 38 successful fusions to the HU values adjacent to 14 nonunions. ${ }^{31}$ There were no differences in age, sex, body mass index, or smoking status between the 2 cohorts. Hounsfield unit measurements both within the fusion construct and throughout the adjacent spine were recorded using a previously described technique, ${ }^{30}$ and mean values between the groups were assessed using a Student t-test.

\section{Results}

\section{Reliability}

The technique described has excellent intraobserver and interobserver reliability, ${ }^{30}$ with interclass correlation coefficients of 0.964 and 0.975 , respectively. This reliability has been confirmed in other investigations. ${ }^{17,25}$ Using the average of 3 measurements at each vertebra compared with a single measurement appears to offer little advantage in terms of reliability. ${ }^{26,27}$ No differences are seen at different levels within the vertebral body (Fig. 2).

\section{Normative Data}

The HU values decrease linearly by decade of life, with a significant correlation observed between age and HU (Table 2; Fig. 3). ${ }^{30}$ The mean HU in females ranged from 250 in young adults to less than 79 in those in their 9th decade. As expected in females, there was a notable decrease in HU around the time of menopause (Fig. 3 left). Normative male data demonstrated a more linear

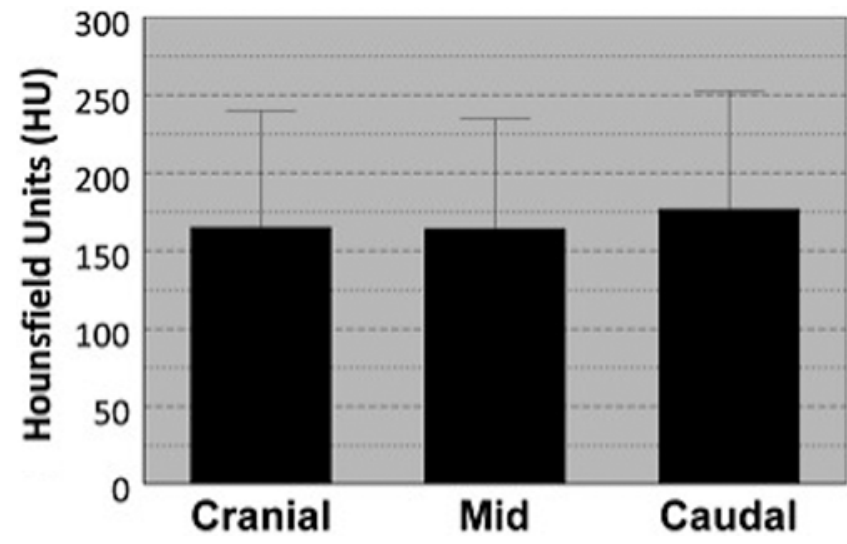

FIG. 2. No differences were observed between HU values obtained at different locations within lumbar vertebral bodies.

decrease in HU with age (Fig. 3 right). The mean HU in males ranged from 270 in young adults to 85 in those in their 9th decade.

These findings were corroborated by a subsequent investigation of 128 patients, which also demonstrated strong correlations between $\mathrm{HU}$ values of the lumbar spine and T-scores from DXA scans, with a trend toward decreasing $\mathrm{HU}$ values with increasing age. ${ }^{18}$

\section{TABLE 2: Normative data of HU values from L-1 to S-1}

\begin{tabular}{cccc}
\hline & \multicolumn{3}{c}{ Mean HU Values } \\
\cline { 2 - 4 } Level & $\begin{array}{c}\text { Meredith et al. } \\
(\mathrm{n}=20)^{*}\end{array}$ & $\begin{array}{c}\text { Schreiber et al. } \\
(\mathrm{n}=80) \dagger\end{array}$ & $\begin{array}{c}\text { Anderson et } \\
\text { al., unpublished } \\
\text { data }(\mathrm{n}=280) \ddagger\end{array}$ \\
\hline T-1 & 213.7 & & 255.3 \\
\hline T-2 & 188.7 & & 237.4 \\
\hline T-3 & 190.9 & & 226.2 \\
\hline T-4 & 165.8 & & 225.1 \\
\hline T-5 & 160.1 & & 225.1 \\
\hline T-6 & 165.0 & & 216.5 \\
\hline T-7 & 169.2 & & 212.6 \\
\hline T-8 & 171.1 & & 207.2 \\
\hline T-9 & 179.4 & & 212.7 \\
\hline T-10 & 163.8 & & 212.7 \\
\hline T-11 & 154.6 & & 222.2 \\
\hline T-12 & 142.3 & & 217.6 \\
\hline L-1 & 149.9 & 170.5 & 236.3 \\
\hline L-2 & 170.4 & 169.2 & 234.3 \\
\hline L-3 & 161.8 & 166.8 & 227.3 \\
\hline L-4 & 175.3 & 167.7 & 229.7 \\
\hline L-5 & 180.5 & & 242.6 \\
\hline S-1 & 209.1 & & \\
\hline
\end{tabular}

* Study group comprised fusion patients ranging in age from 49 to 80 years.

$\dagger$ Study group comprised trauma patients ranging in age from 20 to 80 years.

$\ddagger$ Study group comprised trauma patients ranging in age from 20 to 30 years. 

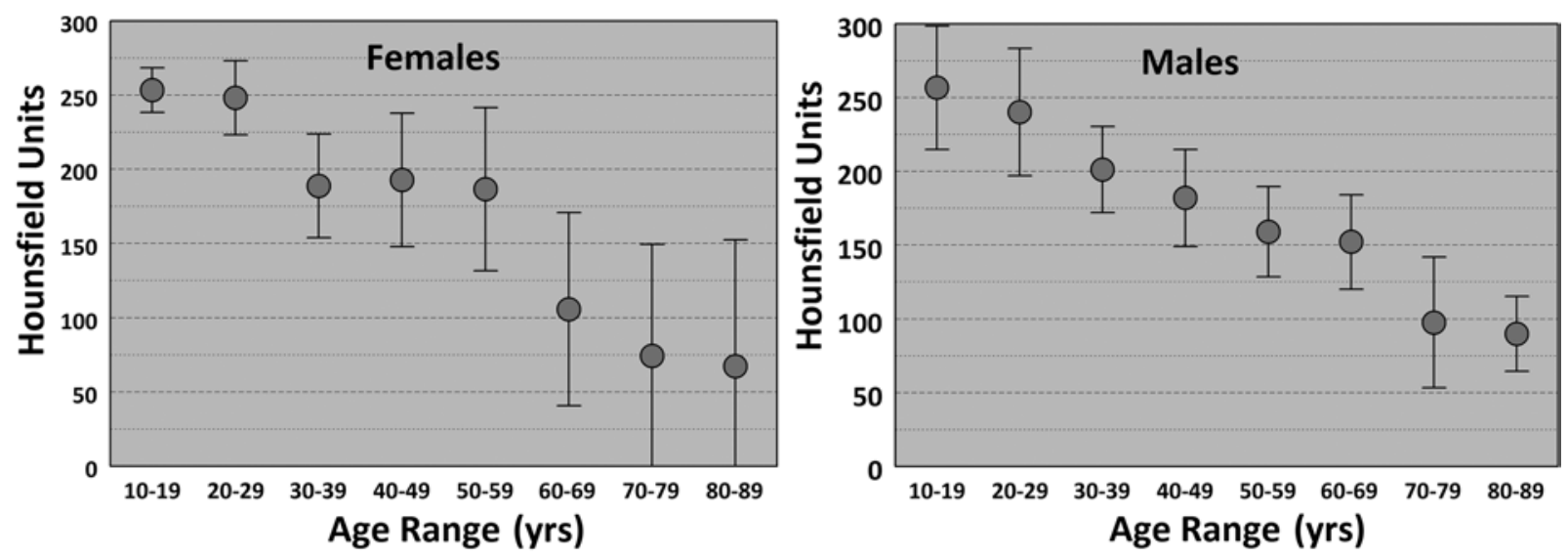

FIG. 3. Normative data of HU values in females (left) and males (right) stratified by decade of life.

The control data from previous studies provide normative data for $\mathrm{HU}$ values from the T-1 vertebral body to the $\mathrm{S}-1$ vertebral body. ${ }^{22,30}$ In addition, we evaluated 280 healthy female patients who underwent thoracic CT scanning to rule out spinal injury after trauma. Normative data, which can be useful for both clinical and research purposes, are shown in Table 2.

\section{Correrlation of HU Value to DXA}

There was good correlation of HU value to DXA for both BMD and T-score. ${ }^{30}$ The Pearson correlation coefficients were 0.44 and 0.48 for BMD and T-score, respectively. The correlation for both parameters was highly statistically significant ( $\mathrm{p}<0.001$, Fig. 4).

\section{Threshold for Diagnosis of Osteoporosis}

Patients were grouped as normal, osteopenic, and osteoporotic based on DXA T-score and the WHO criteria (Fig. 5). ${ }^{30}$ The mean HU values were 133.0 (95\% CI 118.4-147.5) for the normal group, 100.8 (95\% CI 93.1-108.8) for the osteopenia group, and 78.5 (95\% CI 61.9-95.1) for the osteoporotic group (Table 3).

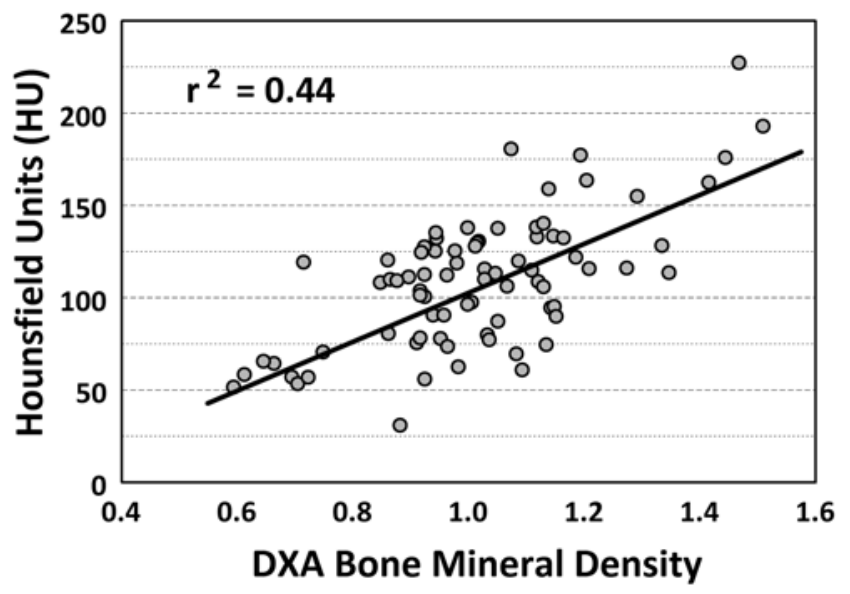

FIG. 4. Correlation between BMD assessed via DXA (in $\mathrm{g} / \mathrm{cm}^{2}$ ) and $\mathrm{HU}$ measurements in the lumbar spine. Adapted with permission from Schreiber JJ et al: J Bone Joint Surg 93:1057-1063, 2011. www.jbjs.org.

\section{HU Value and Fracture Risk}

In the 20 patients with adjacent-segment fracture following spinal fusion surgery, HU measurements at the fractured vertebral body were significantly lower in the fracture group compared with matched controls (145.6 vs 199.4, $\mathrm{p}=0.006) .{ }^{22}$ Global HU measurements throughout the thoracic and lumbar spines were also significantly lower in the fracture group (139.9 vs 170.1, $\mathrm{p}=0.032)$. This study also provided normative data for HU from T-1 to S-1 (Table 2).

\section{HU Value and Fusion Success}

In the investigation assessing the association between HU with fusion success, the successful fusion cohort (n = 38) had significantly higher HU measurements than the nonunion cohort $(\mathrm{n}=14)$ both adjacent to the fusion (203.3 vs $139.8, \mathrm{p}<0.001)$ and globally throughout the noninstrumented spine $(133.7$ vs $107.3, \mathrm{p}<0.05) .{ }^{31}$

\section{Discussion}

When a CT scan has been obtained, HU measurements can easily be obtained by any practitioner and can provide information on both local and global bone density with no additional cost and minimal effort. This in-

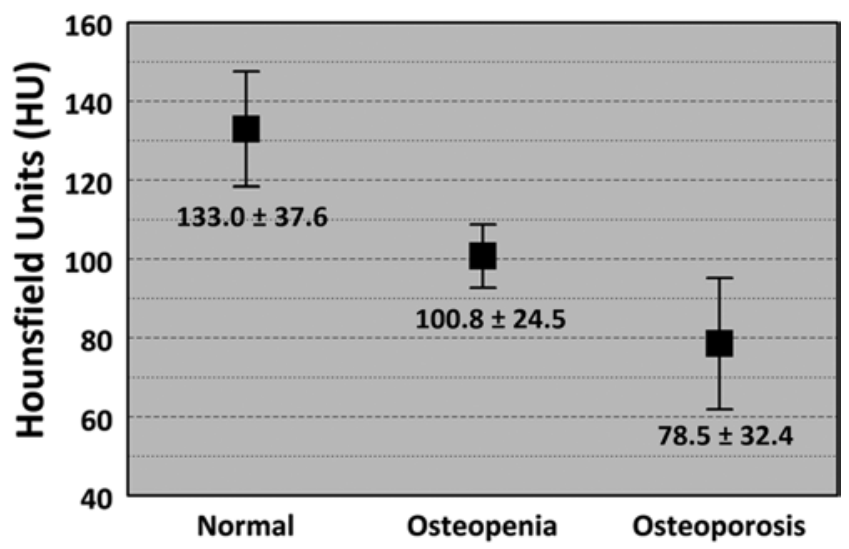

FIG. 5. Mean $\mathrm{HU}$ values $( \pm \mathrm{SD})$ in the lumbar spine representative of normal bone, osteopenia, and osteoporosis. Error bars depict 95\% Cl. 
Use of computed tomography for assessing bone mineral density

TABLE 3: Previously defined means, standard deviations, and $95 \%$ confidence intervals of HU values of the lumbar spine and humeral head in patients meeting WHO criteria for normal, osteopenia, or osteoporosis based on T-scores*

\begin{tabular}{|c|c|c|c|c|c|}
\hline \multirow[b]{2}{*}{ Group } & \multirow[b]{2}{*}{ T-Score } & \multicolumn{2}{|c|}{ Lumbar Spine $\dagger$} & \multicolumn{2}{|c|}{ Humeral Head $\ddagger$} \\
\hline & & Mean \pm SD & $95 \% \mathrm{Cl}$ & Mean \pm SD & $95 \% \mathrm{Cl}$ \\
\hline normal & -1.0 or greater & $133.0 \pm 37.6$ & $118.4-147.5$ & $121.3 \pm 27.9$ & $115.8-126.7$ \\
\hline osteopenia & less than -1.0 or greater than -2.5 & $100.8 \pm 24.5$ & $93.1-108.8$ & $103.4 \pm 26.1$ & $98.2-108.7$ \\
\hline osteoporosis & -2.5 or less & $78.5 \pm 32.4$ & $61.9-95.1$ & $92.1 \pm 29.1$ & $81.0-103.2$ \\
\hline
\end{tabular}

formation can subsequently be used to identify metabolic bone disease and the need for additional imaging or laboratory assessments. In the setting of trauma, this information can provide insight into injury etiology and give the treating surgeon immediate preoperative knowledge of the bone quality that may affect surgical decision making. When appropriate, it may also enable the physician to initiate appropriate treatment or to refer the patient for further management. At this time, we do not believe that CT scanning should be used for screening purposes or as a substitute for DXA, but when already available the data can be useful to identify at-risk patients.

While BMD has historically been assessed using DXA, the HU values from CT scans offer several benefits. Hounsfield unit values can be obtained throughout the entire spine where DXA standards have not been defined. Additionally, DXA results can be artificially elevated by both lumbar scoliosis and degenerative changes. ${ }^{24,28}$ Other advantages of HU assessment over DXA assessment include the fact that HU assessment represents 3D $\mathrm{BMD}$ and that can be localized to any ROI.

\section{HU Value and Osteoporosis}

Osteoporosis is a relevant issue in the field of spine surgery, as over half of all females and 1 in 6 males undergoing spine surgery are osteoporotic according to their DXA scanning findings and based on World Health Organization definitions..$^{5}$ Despite the prevalence, a survey of spine surgeons recently found that only $22 \%$ performed DXA and only $11 \%$ checked metabolic laboratory values prior to performing noninstrumented fusion. ${ }^{7}$ Multiple studies have explored the ability of diagnostic CT scanning to identify osteopenia and osteoporosis both based on HU values obtained from the spine ${ }^{30,34}$ and proximal humerus. ${ }^{25}$ With an estimated 62 million CT scanning studies being performed annually in the US, the information provided by these studies is oftentimes available to the practitioner at no additional cost and can provide invaluable assessments of the patient's local and global bone quality.

Several methods have been used to correlate the diagnosis of osteopenia and osteoporosis based on HU and DXA. One method is to group patients based on DXA and calculate mean HU and $95 \%$ confidence intervals. Studies by Schreiber et al. ${ }^{30}$ and Pervaiz et al. ${ }^{25}$ found similar values for normal, osteopenic, and osteoporotic patients in the spine and proximal humerus (Table 3). An impor- tant threshold based on these studies is an HU value of less than 120, which indicates likely bone insufficiency and suggests the need for further investigation (Fig. 6). In the case shown in Fig. 6, not only was diffuse osteoporosis suggested, but primary screening for a cause of the osteoporosis resulted in a diagnosis of multiple myeloma.

Another method is to determine cutoff values that optimize sensitivity and specificity using receiver operating characteristic analysis. Pickhardt et al. compared CTderived HU to DXA results in a series of 1867 patients and found that an HU threshold of 160 or less at L-1 was 90\% sensitive for distinguishing osteoporosis from osteopenia, while an HU value of 110 was $90 \%$ specific. ${ }^{27}$

\section{HU Value and Spinal Fracture Risk}

The correlation between bone density and fracture risk is well established, ${ }^{37}$ with subsequent studies showing strong correlations between HU measurements and bone density. ${ }^{18,25-27,30}$ The proof of concept that HU values can be used be used to estimate regional bone strength and fracture risk was first established using polyurethane foam, where a linear correlation was found between HU value and compressive strength across densities representative of human bone. ${ }^{30}$

Estimation of fracture risk is a key variable in determining whether patients should be treated for metabolic bone disease. Several clinical factors other than BMD account for fracture risk, ${ }^{14}$ which led to the development of the Fracture Risk Assessment Tool (FRAX). ${ }^{15}$ This tool calculates the 10-year probability of a major osteoporotic fracture while incorporating the following factors: age, sex, race, height, weight, BMI, history of fragility fracture, family history, use of oral glucocorticoids, rheumatoid arthritis and other secondary causes of osteoporosis, current smoking status, alcohol intake, and geographic area.

The ability of HU values to predict fracture risk has been investigated. Meredith et al. found an association between HU measurements and adjacent-segment fractures after spinal fusion..$^{22}$ The authors concluded that HU measurements provide a promising tool for assessing adjacent-segment fracture risk after spinal fusion.

Additionally, in a prospective study of 132 patients treated with vertebroplasty for osteoporotic compression fractures, Liu et al. found that decreased HU values of nonfractured vertebral bodies were a significant risk factor for subsequent compression fracture $(\mathrm{p}=0.001){ }^{20}$ 

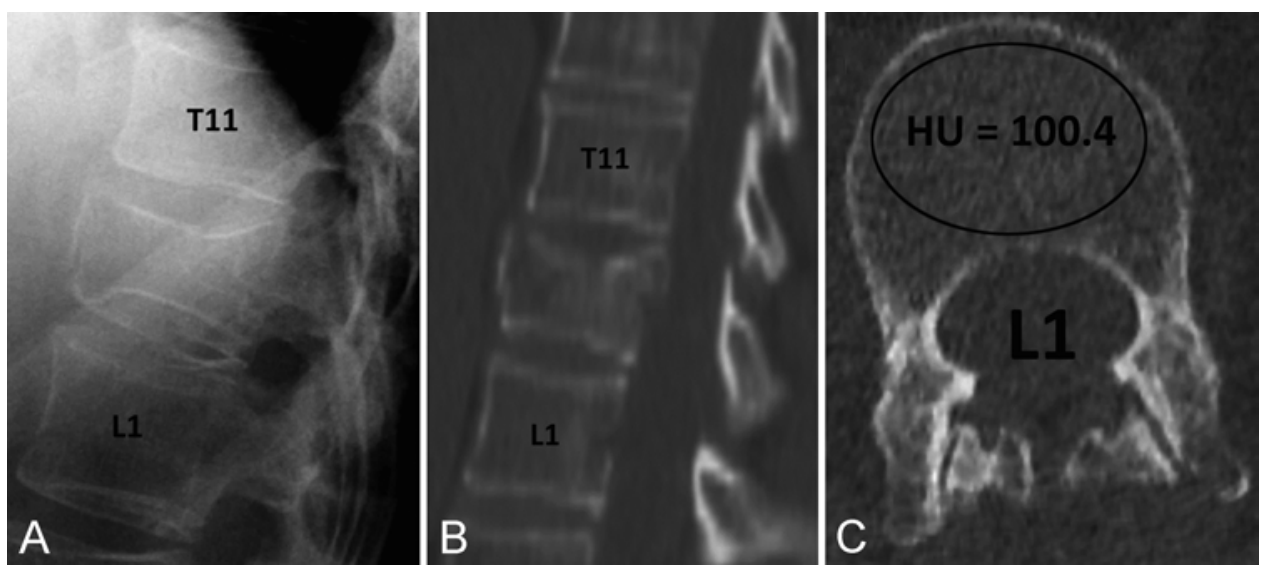

FIG. 6. A 68-year-old man fell while skiing in Colorado, sustaining a T-12 burst fracture. A: Lateral radiograph shows demineralization and compression of both anterior and middle columns. B: Sagittal CT demonstrating burst fracture with minimum kyphosis of T-12. Demineralization is present. C: Hounsfield units were determined for an elliptical ROI that measured 100.4, indicating probable osteopenia or osteoporosis. The DXA value of the femoral neck was -1.4 , confirming osteopenia. An evaluation for primary causes of osteoporosis was positive for Bence-Jones proteins and monoclonal gammopathy. Bone marrow pathology was positive for multiple myeloma, which presumably was responsible for the bone demineralization.

The 36 patients who developed subsequent compression fractures had a mean vertebral $\mathrm{HU}$ value of 55, which would be categorized as osteoporotic based on previously defined ranges (Table 3)..$^{30}$

\section{HU Value and Spinal Fusion}

More recently, the ability of HU values to serve as a prognostic tool for predicting success of lumbar spine fusion was assessed. ${ }^{31}$ The HU measurements of bone graft within intervertebral lumbar cages have been longitudinally studied as a marker of biological activity in the postoperative period and have shown predictable timedependent changes indicative of graft incorporation. ${ }^{33}$

\section{HU Value and Durotomy Risk}

Incidental durotomy during spinal surgery can result in postoperative sequelae of pseudomeningocele, dural cutaneous fistula formation, or infection if not appropriately recognized and repaired, whereas postrepair management with recumbency and bed rest can increase hospital stay duration and associated costs. ${ }^{4,8}$ The association between HU measurement and incidental durotomy was recently investigated. ${ }^{8}$ Given the link between dural cells and osteoblast development, ${ }^{32} \mathrm{Du}$ et al. proposed that patients with lower bone density may have structurally attenuated dura, thereby predisposing them to a higher risk of incidental durotomy. In this series, 71 patients with an incidental durotomy following spinal surgery were matched with controls, and HU was significantly lower in the durotomy group (149 vs $177, \mathrm{p}=0.023$ ). An HU value of 169 optimized sensitivity (72\%) and specificity (45\%), whereas patients with values below this threshold were twice as likely to have an incidental durotomy (OR 2.092, $\mathrm{p}=0.037) .{ }^{8}$

\section{HU Value in Lumbar Trauma}

In the setting of lumbar trauma, HU assessment of regional bone quality can provide insight into underlying pathology that may have contributed to the injury. An example case is illustrated in Fig. 6, which depicts a T-12 burst fracture that occurred in a 68 -year-old man who fell while skiing. Local demineralization was visually apparent, and $\mathrm{HU}$ measurements suggested an underlying pathology of poor bone quality. Further workup resulted in a diagnosis of multiple myeloma, presumably contributing to the patient's injury pattern. We have found that approximately $20 \%$ of patients older than 50 years with traumatic spine fractures have occult osteoporosis based on simple HU measurements.

In the trauma setting, HU measurements can also predict success and failure of surgical instrumentation. The HU values of local bone quality have been shown to not only affect fracture risk ${ }^{17}$ but can also prognosticate implant stability ${ }^{25}$ and hardware failure. ${ }^{16}$ When instrumentation is required, the included levels chosen as well as technical modifications can be influenced by knowledge of local and global bone quality.

\section{Preoperative Surgical Planning}

Preoperative knowledge of poor bone quality can affect surgical planning in both traumatic and degenerative cases. Technical modifications to spine surgery intended to prevent fracture or loosening of hardware in osteoporotic bone can be implemented, such as increasing pedicle screw size, using conical screws, undertapping the screw track, utilizing coated or expandable screws, or the application of injectable fillers at the operative site or adjacent vertebral level. ${ }^{2,6,12,23,29}$ Given that HU values correlate with risk of fracture and proximal junctional kyphosis after fusion, ${ }^{22}$ changing fusion levels based on preoperative HU measurements may be warranted. Unfortunately, specific recommendations regarding the algorithm for alternatives based on HU values are lacking and need further research.

\section{Future Directions}

While many associations have been found between $\mathrm{HU}$ values and BMD, compressive strength, fracture risk, 


\section{Use of computed tomography for assessing bone mineral density}

and fusion, more prospectively designed studies are required to truly appreciate the utility of HU values in assessing bone quality, assist in surgical decision making, and prognosticate outcomes of fusion success, fracture risk, and implant stability.

Given the multitude of correlations described between HU and bone quality, it would seem likely that preoperative HU assessment of local and global bone quality could be used for planning spinal instrumentation. While future studies are needed to answer this question in the spine, local HU values have been used in the field of dentistry for implant planning. In both cadaver and in vivo studies of the mandible and maxilla, HU values have been used to assess regional bone density and have been correlated with insertional torque and implant stability. ${ }^{35,36}$

\section{Conclusions}

The correlation between HU measurement and BMD has been firmly established. With an aging population and increasing prevalence of metabolic bone disease, the treating surgeon must be of aware of the perioperative effects of poor bone quality. In the trauma setting, this can illuminate contributing factors to the injury and affect intraoperative techniques and the risk of postoperative complications. When CT scans have been obtained as a routine measure in preoperative evaluations, they may contain invaluable information of regional and global bone quality at no additional cost. This information can be useful for appropriate patient counseling regarding perioperative risks, or it can be used to identify the need for additional studies, appropriate referral, or treatment initiation for osteoporosis.

\section{Disclosure}

The authors report no conflict of interest concerning the materials or methods used in this study or the findings specified in this paper.

Author contributions to the study and manuscript preparation include the following. Conception and design: Anderson, Schreiber. Acquisition of data: Anderson, Schreiber. Analysis and interpretation of data: Anderson, Schreiber. Drafting the article: all authors. Critically revising the article: all authors. Reviewed submitted version of manuscript: all authors. Approved the final version of the manuscript on behalf of all authors: Anderson. Statistical analysis: Anderson, Schreiber. Administrative/technical/material support: Anderson, Schreiber. Study supervision: all authors.

\section{References}

1. Assessment of fracture risk and its application to screening for postmenopausal osteoporosis. Report of a WHO Study Group. World Health Organ Tech Rep Ser 843:1-129, 1994

2. Brantley AG, Mayfield JK, Koeneman JB, Clark KR: The effects of pedicle screw fit. An in vitro study. Spine (Phila Pa 1976) 19:1752-1758, 1994

3. Burge R, Dawson-Hughes B, Solomon DH, Wong JB, King A, Tosteson A: Incidence and economic burden of osteoporosisrelated fractures in the United States, 2005-2025. J Bone Miner Res 22:465-475, 2007

4. Cammisa FP Jr, Girardi FP, Sangani PK, Parvataneni HK, Cadag S, Sandhu HS: Incidental durotomy in spine surgery. Spine (Phila Pa 1976) 25:2663-2667, 2000
5. Chin DK, Park JY, Yoon YS, Kuh SU, Jin BH, Kim KS, et al: Prevalence of osteoporosis in patients requiring spine surgery: incidence and significance of osteoporosis in spine disease. Osteoporos Int 18:1219-1224, 2007

6. Cook SD, Salkeld SL, Whitecloud TS III, Barbera J: Biomechanical evaluation and preliminary clinical experience with an expansive pedicle screw design. J Spinal Disord 13:230236,2000

7. Dipaola CP, Bible JE, Biswas D, Dipaola M, Grauer JN, Rechtine GR: Survey of spine surgeons on attitudes regarding osteoporosis and osteomalacia screening and treatment for fractures, fusion surgery, and pseudoarthrosis. Spine $\mathbf{J}$ 9:537-544, 2009

8. Du JY, Aichmair A, Ueda H, Girardi FP, Cammisa FP, Lebl DR: Vertebral body Hounsfield units as a predictor of incidental durotomy in primary lumbar spinal surgery. Spine (Phila Pa 1976) 39:E593-E598, 2014

9. Eswaran SK, Gupta A, Adams MF, Keaveny TM: Cortical and trabecular load sharing in the human vertebral body. J Bone Miner Res 21:307-314, 2006

10. Goodsitt MM, Christodoulou EG, Larson SC, Kazerooni EA: Assessment of calibration methods for estimating bone mineral densities in trauma patients with quantitative CT: an anthropomorphic phantom study. Acad Radiol 8:822-834, 2001

11. Hart RA, Prendergast MA: Spine surgery for lumbar degenerative disease in elderly and osteoporotic patients. Instr Course Lect 56:257-272, 2007

12. Hu SS: Internal fixation in the osteoporotic spine. Spine (Phila Pa 1976) 22 (24 Suppl):43S-48S, 1997

13. Johnell O, Kanis JA: An estimate of the worldwide prevalence and disability associated with osteoporotic fractures. Osteoporos Int 17:1726-1733, 2006

14. Kanis JA: Diagnosis of osteoporosis and assessment of fracture risk. Lancet 359:1929-1936, 2002

15. Kanis JA, Johnell O, Oden A, Johansson H, McCloskey E: FRAX and the assessment of fracture probability in men and women from the UK. Osteoporos Int 19:385-397, 2008

16. Krappinger D, Bizzotto N, Riedmann S, Kammerlander C, Hengg C, Kralinger FS: Predicting failure after surgical fixation of proximal humerus fractures. Injury 42:1283-1288, 2011

17. Krappinger D, Roth T, Gschwentner M, Suckert A, Blauth M, Hengg C, et al: Preoperative assessment of the cancellous bone mineral density of the proximal humerus using CT data. Skeletal Radiol 41:299-304, 2012

18. Lee S, Chung CK, Oh SH, Park SB: Correlation between bone mineral density measured by dual-energy X-Ray absorptiometry and Hounsfield units measured by diagnostic CT in lumbar spine. J Korean Neurosurg Soc 54:384-389, 2013

19. Link TM, Koppers BB, Licht T, Bauer J, Lu Y, Rummeny EJ: In vitro and in vivo spiral CT to determine bone mineral density: initial experience in patients at risk for osteoporosis. Radiology 231:805-811, 2004

20. Liu WG, He SC, Deng G, Guo JH, Fang W, Zhu GY, et al: Risk factors for new vertebral fractures after percutaneous vertebroplasty in patients with osteoporosis: a prospective study. $\mathbf{J}$ Vasc Interv Radiol 23:1143-1149, 2012

21. Marshall D, Johnell O, Wedel H: Meta-analysis of how well measures of bone mineral density predict occurrence of osteoporotic fractures. BMJ 312:1254-1259, 1996

22. Meredith DS, Schreiber JJ, Taher F, Cammisa FP Jr, Girardi FP: Lower preoperative Hounsfield unit measurements are associated with adjacent segment fracture after spinal fusion. Spine (Phila Pa 1976) 38:415-418, 2013

23. Ono A, Brown MD, Latta LL, Milne EL, Holmes DC: Triangulated pedicle screw construct technique and pull-out strength of conical and cylindrical screws. J Spinal Disord 14:323-329, 2001

24. Pappou IP, Girardi FP, Sandhu HS, Parvataneni HK, Cam- 
misa FP Jr, Schneider R, et al: Discordantly high spinal bone mineral density values in patients with adult lumbar scoliosis. Spine (Phila Pa 1976) 31:1614-1620, 2006

25. Pervaiz K, Cabezas A, Downes K, Santoni BG, Frankle MA: Osteoporosis and shoulder osteoarthritis: incidence, risk factors, and surgical implications. J Shoulder Elbow Surg 22:e1-e8, 2013

26. Pickhardt PJ, Lee LJ, del Rio AM, Lauder T, Bruce RJ, Summers RM, et al: Simultaneous screening for osteoporosis at CT colonography: bone mineral density assessment using MDCT attenuation techniques compared with the DXA reference standard. J Bone Miner Res 26:2194-2203, 2011

27. Pickhardt PJ, Pooler BD, Lauder T, del Rio AM, Bruce RJ, Binkley N: Opportunistic screening for osteoporosis using abdominal computed tomography scans obtained for other indications. Ann Intern Med 158:588-595, 2013

28. Rand T, Seidl G, Kainberger F, Resch A, Hittmair K, Schneider $\mathrm{B}$, et al: Impact of spinal degenerative changes on the evaluation of bone mineral density with dual energy X-ray absorptiometry (DXA). Calcif Tissue Int 60:430-433, 1997

29. Sandén B, Olerud C, Larsson S: Hydroxyapatite coating enhances fixation of loaded pedicle screws: a mechanical in vivo study in sheep. Eur Spine J 10:334-339, 2001

30. Schreiber JJ, Anderson PA, Rosas HG, Buchholz AL, Au AG: Hounsfield units for assessing bone mineral density and strength: a tool for osteoporosis management. J Bone Joint Surg Am 93:1057-1063, 2011

31. Schreiber JJ, Hughes AP, Taher F, Girardi FP: An association can be found between Hounsfield units and success of lumbar spine fusion. HSS J 10:25-29, 2014

32. Spector JA, Greenwald JA, Warren SM, Bouletreau PJ, Crisera FE, Mehrara BJ, et al: Co-culture of osteoblasts with immature dural cells causes an increased rate and degree of
J. J. Schreiber, P. A. Anderson, and W. K. Hsu

osteoblast differentiation. Plast Reconstr Surg 109:631-644, 2002

33. Spruit M, Meijers H, Obradov M, Anderson PG: CT density measurement of bone graft within an intervertebral lumbar cage: increase of Hounsfield units as an indicator for increasing bone mineral content. J Spinal Disord Tech 17:232-235, 2004

34. Tay WL, Chui CK, Ong SH, Ng AC: Osteoporosis screening using areal bone mineral density estimation from diagnostic CT images. Acad Radiol 19:1273-1282, 2012

35. Turkyilmaz I, Aksoy U, McGlumphy EA: Two alternative surgical techniques for enhancing primary implant stability in the posterior maxilla: a clinical study including bone density, insertion torque, and resonance frequency analysis data. Clin Implant Dent Relat Res 10:231-237, 2008

36. Turkyilmaz I, Sennerby L, McGlumphy EA, Tözüm TF: Biomechanical aspects of primary implant stability: a human cadaver study. Clin Implant Dent Relat Res 11:113-119, 2009

37. Unnanuntana A, Gladnick BP, Donnelly E, Lane JM: The assessment of fracture risk. J Bone Joint Surg Am 92:743-753, 2010

Manuscript submitted March 8, 2014

Accepted May 7, 2014.

Please include this information when citing this paper: DOI: 10.3171/2014.5.FOCUS1483.

Address correspondence to: Paul A. Anderson, M.D., Department of Orthopedics \& Rehabilitation, University of Wisconsin, UWMF Centennial Bldg., 1685 Highland Ave., 6th Floor, Madison, WI 53705.email: anderson@ortho.wisc.edu. 